\title{
Laboreal
}

Volume $9 \mathrm{~N}^{\circ} 2$ | 2013

Varia

\section{Movilidades, territorios y servicio público : debates sobre el interés colectivo al margen del paradigma de una sociedad móvil}

Mobilidades, territórios e serviço público : debates sobre o interesse colectivo à margem do paradigma de uma sociedade móvel

Mobilités, territoires et service public : débats à propos de l'intérêt collectif à la marge du paradigme d'une société mobile

Mobilities, territories and public service: debates over the collective interest at the margins of the paradigm of a mobile society

\section{Liliana Cunha}

Traductor: Rossana Cacivio.

\section{(2) OpenEdition \\ Journals}

Edición electrónica

URL: http://journals.openedition.org/laboreal/5740

DOI: $10.4000 /$ laboreal. 5740

ISSN: 1646-5237

Editor

Universidade do Porto

Referencia electrónica

Liliana Cunha, « Movilidades, territorios y servicio público : debates sobre el interés colectivo al margen del paradigma de una sociedad móvil », Laboreal [En línea], Volume 9 №2 | 2013, Publicado el 01 diciembre 2013, consultado el 24 septiembre 2020. URL : http://journals.openedition.org/laboreal/ 5740 ; DOI : https://doi.org/10.4000/laboreal.5740

Este documento fue generado automáticamente el 24 septiembre 2020.

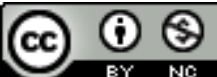

Laboreal está licenciado com uma Licença Creative Commons - Atribuição-NãoComercial 4.0 Internacional. 


\section{Movilidades, territorios y servicio público : debates sobre el interés colectivo al margen del paradigma de una sociedad móvil}

Mobilidades, territórios e serviço público : debates sobre o interesse colectivo à margem do paradigma de uma sociedade móvel

Mobilités, territoires et service public : débats à propos de l'intérêt collectif à la marge du paradigme d'une société mobile

Mobilities, territories and public service: debates over the collective interest at the margins of the paradigm of a mobile society

\section{Liliana Cunha}

Tradución : Rossana Cacivio.

\section{REFERENCIA}

Cunha, L. (2012). Mobilidades, territórios e serviço público : debates sobre o interesse colectivo à margem do paradigma de uma sociedade móvel. Tese apresentada para efeitos de obtenção do grau de Doutor em Psicologia. Porto : FPCE-UP.

\section{NOTA DEL EDITOR}

Manuscrito recibido en : Septiembre/2013

Aceptado tras peritaje : Octubre/2013 


\section{Introducción}

1 Esta tesis sitúa su análisis en el contexto de la movilidad en el transporte público, tratando de comprenderla a partir de la caracterización del sistema de transporte, de las opciones de organización del trabajo, del hacer y desde el punto de vista de los actores que la conciben (diseñadores de redes de movilidad) y producen (conductores).

2 Enmarcada en la tradición científica de la ergonomía y de la psicología del trabajo de la actividad, y articulada con la influencia de la "perspectiva ergológica", la tesis discute la contribución de la actividad de los conductores del sector del transporte colectivo de pasajeros (transporte en ómnibus), en la compensación de las desigualdades sociales y territoriales que los sistemas de transporte son susceptibles de reproducir.

3 Se fundamenta así el uso privilegiado del concepto de movilidad a el de transporte, a menudo confundidos e invocados indiscriminadamente. Si el transporte se entiende frecuentemente, desde una lógica funcionalista, como lo desplazamiento entre un origen y un destino predefinido y por lo tanto independiente de sus actores, el uso del concepto de movilidad se manifiesta como una producción social plural, modelada por la historia (donde también interviene la actividad del trabajo de cada uno), por las características de los territorios y por la acción pública. En este sentido, el pasaje de transporte a movilidad desde nuestra perspectiva, es la manifestación de la actividad humana, y más específicamente de la actividad del trabajo. De lo contrario, ¿cómo podría concretarse el ansiado ajuste entre la demanda de servicios de transporte público y su oferta, de un modo "desencastrado" [1] de las realidades concretas y sin considerar las mediaciones sociales que también los determinan?

\section{2. "Ser móvil" : valor estructurante de la posmodernidad}

Los discursos sobre la sociedad contemporánea sostienen la movilidad como un "valor dominante y transversal (...) [y con un] carácter fundamentalmente positivo" (Lannoy \& Ramadier, 2007, p. 12, traducción libre), de tal forma que hoy hablamos de una "movilidad generalizada" (ídem), o incluso de una "ideología mobilitária" (Mincke \& Montulet, 2010), consustanciadas con la afirmación de una disolución de las restricciones espaciales, en favor de la conquista de la libertad (individual) de circulación.

Pero si se reconoce la influencia de los fenómenos de individuación en la construcción de las sociedades contemporáneas, y una tendencia a la institución de formas de movilidad acordes con esto, las llamadas "automovilidades" (Urry, 2004), ¿cual es el espacio para reflexionar sobre la movilidad colectiva y sobre los proyectos de "socialización de las condiciones de circulación" ? (Castells, 2000, p. 280, traducción libre).

6 Aunque hoy en día la movilidad en transporte público, y más concretamente en ómnibus, sea connotada como "pre-moderna" (al imponer límites al proyecto de una movilidad sin fronteras espaciales ni temporales), en comparación con la movilidad individual "moderna" (Mom, 2009), asumimos que su análisis e intervención no puede ser residual, temiendo contribuir a la exclusión de ciertas categorías de la población y a 
ciertos espacios, mantenidos al margen de la hegemonía de este paradigma de una "sociedad móvil".

\subsection{Un "derecho a la movilidad" consagrado}

7 A pesar de que la movilidad no es homogénea, ni garantizada para todos, en todas partes, el "derecho a la movilidad", y en particular a la movilidad en transporte público, se reconoce como una especie de derecho "genérico" que determina la eficacia de la mayoría de los otros derechos" (Ascher, 2010, p. 197, traducción libre). Su garantía es una condición de participación en la vida social, para el acceso a la educación, salud, empleo y otras actividades y servicios, por lo que se afirma igualmente como condición de inclusión social y de ciudadanía, justificando la acción pública en esta área.

En efecto, este derecho está consagrado en el Tratado constitutivo de la Comunidad Europea (TCE) en forma de una "libertad de movimiento". En Portugal, la Ley de Bases del Sistema de Transportes Terrestres (Ley $n^{\circ}$ 10/90, de 17 de marzo), tal derecho se expresa como "libertad de elección de los medios de transporte, incluyendo el uso del transporte por cuenta propia" y "proporcionado a los usuarios en paridad de condiciones, igualdad de tratamiento en el acceso y disfrute de estos servicios" (artículo $2^{\circ}, \mathrm{n}^{\mathrm{0}} 2$, traducción libre).

De acuerdo con la perspectiva ergológica, este derecho podría encuadrarse en las "normas precedentes" formalmente instituidas, con el propósito de regular la vida colectiva y la protección del interés público (Polo de la Politeia), a los que estarían asociados "valores sin dimensión" (Schwartz, 2000a).

10 Es en este intento de garantizar el interés general, suponiendo que el suministro de servicios de transporte sea susceptible de reproducir ciertas desigualdades (mayor oferta en lugares marcados por una alta densidad poblacional, por ejemplo), que se reconoce la actividad en este sector como "servicio público", aunque no exento del debate de valores que atraviesa la evolución de este concepto y sus prácticas.

\subsection{La movilidad como matriz institucional : el "servicio público" de transportes}

11 La tradición clásica del servicio público es a menudo connotada como la asunción por parte del poder público, de las atribuciones que tienen por objeto satisfacer las necesidades de interés general (Chevallier, 1987), y que no son susceptibles de ser satisfechas por una economía de mercado (Le Van-Lemesle, 2005), o por lo menos de forma equivalente.

Sin embargo, la entrada en los años 90 del siglo XX protagonizó una crisis conceptual de la noción de servicio público (Nemery \& Routier, 1999), en particular, con la privatización de los servicios públicos, como sucedió en el sector de transporte colectivo de pasajeros por carretera en Portugal (Cunha \& Lacomblez, 2007; 2010 ; Scheller, Cunha, Nogueira \& Lacomblez, 2013). El contenido de la noción de servicio público de hoy es quizás menos obvio y menos consensual. Esta idea fue ampliamente apoyada por Marcello Caetano (citado por Fernández, 1996) afirmando que "el concepto de servicio público es ambigua, tanto en la doctrina como en la práctica" (p.390, traducción libre). 
13 Según Dreyfus (1999), uno de los factores que han sido determinantes en la crisis de los servicios públicos ha sido el "cambio ideológico y cultural" (p.16, traducción libre), tributarias de la libre competencia, la desregulación neoliberal y el desmantelamiento del Estado de bienestar. Además de la crítica sostenida por el enfoque neoliberal, que considera que el mercado puede resolver las necesidades de la mayoría de las personas y que cada uno, según sus opciones, puede en última instancia, definir las dimensiones del servicio que deben ser privilegiadas por las empresas prestatarias, la intervención pública sería sólo necesaria para facilitar el acceso al mercado de aquellos que de otra manera podrían ser excluidos - por ejemplo, por encontrarse en una zona geográfica menos atractiva, o por expresar necesidades que no pueden ser satisfechas en función de los criterios de rentabilidad definidos por los prestadores del servicio. En este sentido, se ha producido una retracción del papel del Estado, la depreciación del sector público y una revalorización del sector privado. En efecto, el Tratado constitutivo de la Comunidad Europea, en su artículo 295, sostiene una posición de "neutralidad sobre el régimen de propiedad en los Estados miembros", lo que significa que la Comunidad defiende el trato igualitario de las empresas encargadas de misiones de interés general, independientemente de su carácter público o privado.

14 Ahora bien, si la prestación de un servicio público, tal como es actualmente concebido, prevé la conciliación de la orientación al mercado con una orientación hacia el bien común, ¿como se construye un compromiso de equilibrio en una situación en que la prestación del servicio público de transporte es al mismo tiempo atravesado por situaciones de competencia entre diferentes operadores [2] ? ¿Cuál es el impacto sobre la actividad del prestador de servicios, en este caso los conductores?

\section{Primer estudio de caso : la actividad de servicio público de los conductores en una línea concesionada por diferentes operadores}

15 Un primer estudio de caso incluyó el análisis de la actividad de los conductores de una línea urbana, la cual se encuentra en la periferia del centro urbano de la ciudad de Porto. Se trata de una línea cuya concesión es compartida por tres operadores diferentes, dos de los cuales son privados y uno público (el trazado de la línea es especialmente común para los dos operadores privados - el origen, el destino y las paradas son también comunes - recorriendo el operador público cerca de la mitad de la ruta compartida con estos operadores).

16 El análisis de la actividad se desarrolló en dos etapas, que corresponden a diferentes fases de la historia de concesión de esta línea de ómnibus. Los objetivos inherentes a la operación del recorrido para cada uno de los operadores eran, en un primer momento del estudio (noviembre de 2003 hasta abril 2004), claramente diferenciados, y nuestro análisis recayó principalmente en un de los operadores privados, que tenía asignada una misión de servicio público, como resultado de un "acuerdo de concesión", establecido con el operador público (Cunha \& Lacomblez, 2006). Sucintamente, para que podamos establecer la prioridad de cada operador: prestar un servicio público de transporte ; cumplir con el "acuerdo de explotación", que se define en base al pago de un valor/km recorrido y por lo tanto, la prioridad es recorrer los kilómetros previstos, a la hora prescrita (operador privado con acuerdo de concesión) ; y llevar el número 
máximo de pasajeros, ya que el ingreso obtenido provenía de la venta de boletos (según el operador privado).

17 Las observaciones revelaron que en contexto real, en lugar de la competencia a la que podríamos pensar que conduciría esta situación de co-actividade (una misma línea y horarios muy próximos), se identificaron estrategias que corresponden a compromisos construidos y validados de forma colectiva. Una de estas estrategias, por ejemplo, consistía en permitir que los conductores del segundo operador privado iniciaran la actividad siempre unos minutos más temprano, ganando una ventaja relativa a los conductores de las otras dos empresas, por la oportunidad de transportar más pasajeros y generar más ingresos, tal como promovían los objetivos definidos para su actividad de trabajo. Los otros conductores incluso facilitaron el logro de este objetivo, tratando de no adelantarse cuando se aproximaban a ellos o permitiéndoles pasarlos, para que continuaran el recorrido delante de ellos.

18 La esencia de este compromiso se construyó en la historia de concesión compartida y se mantuvo debido a que los objetivos relacionados con la actividad de los conductores de cada empresa, al no ser incompatibles ni competitivos, lo permitió. Esta situación explicita el resultado de una negociación compartida dentro de lo que la perspectiva ergológica define como "entidades colectivas relativamente pertinentes" (ECRP) (Schwartz \& Durrive, 2003). La configuración de estas ECRP es espontánea, su circunscripción es fluida, y son vulnerables a los cambios que se van introduciendo en los contextos de trabajo. Sin embargo, estas se constituyen en el marco de valores compartidos y con el fin de regular la "vida en común" en el recorrido, que también se refleja en la mejora del servicio de transporte proporcionado. De hecho, si los conductores hubieran tenido horarios prescriptos muy similares entre sí, los pasajeros aparentemente habrían perdido la ventaja de tener dos o tres alternativas de transporte para el mismo destino. La frecuencia de horarios entre las tres empresas de ómnibus que se proponía introducir en un contexto real, con estrategias colectivas desarrolladas por los conductores, correspondía a un escalonamiento del pasaje de los ómnibus y por lo tanto a una mayor frecuencia en la oferta de transporte.

19 El regreso al terreno, en un segundo momento (mayo de 2007 hasta febrero 2008), se llevó a cabo en un período de cambio en la concesión de esta línea - que muestra que las normas precedentes no son atemporales, aunque reclamen siempre una anticipación de la acción - si bien el trazado del recorrido no había cambiado, y se hubieran mantenido los mismos operadores responsables por su utilización. Además de los cambios en el sistema global de transporte en el área metropolitana de Porto, a raíz de la integración con la red de metro, que tuvo su impacto en la venta de boletos [3], también sufrieron alteraciones las condiciones del "acuerdo de concesión" entre el operador público y el operador privado, cuya actividad analizamos de manera más sistemática. El sistema de compensación de este operador privado, por la asunción de obligaciones de servicio público, se convirtió en la adquisición de derechos sobre los ingresos obtenidos. Sabiendo que siempre hay reverberancias entre las normas antecedentes y la actividad, ¿Que debate de normas protagoniza, en este contexto, la actividad actual de los conductores?

20 Con este cambio, los objetivos de los dos operadores privados se volvieron en competidores. Las entrevistas realizadas a los conductores de la empresa privada en análisis, revelaron que se habían definido nuevas opciones de organización del trabajo, aunque la misión de servicio público atribuida no lo previa. Los horarios fueron 
alterados, buscando garantizar una ventaja comparativa en relación con el segundo operador privado, que con el tiempo enfrentó a los conductores en un conflicto por la "elección" del horario a cumplir (¿el horario establecido por la empresa, o el horario comunicado a los clientes ?) ; se hizo una selección de los conductores para esta línea, entre otras estrategias que han fomentado la competencia entre los conductores de las dos empresas. La actividad es, desde entonces, vivida de una forma dramática y como fuente de sufrimiento por la competencia que comprometió la preservación de este "colectivo" (un colectivo no predefinido, sino constituido por la actividad en un momento dado) ; para sólo "trabajar por la caja" (énfasis en situaciones de competencia y en las inflexiones de orientación mercantil) ; y por la amenaza de una pedagogía de lo que los clientes pueden esperar/exigir de un servicio público de transportes.

El análisis de esta actividad de servicio público hace visible diferentes injunciones contradictorias que se impone (re)conocer. El énfasis en el cumplimiento de las obligaciones inherentes a la noción de servicio público no es de por sí suficiente, es necesario tener en cuenta en qué medida las opciones de organización del trabajo definidos dentro del ámbito de este estatuto, favorecen o, por el contrario, requieren de los trabajadores hacer arbitrajes entre los intereses de la empresa y el interés público (Linhart, 2009). Dicho de otra manera, si se asume un principio de neutralidad relativa al estatuto de las empresas que prestan un servicio público, este principio no puede esquivar la cuestión de las condiciones en que este es co-producido por los actores involucrados, y su impacto en la salud de los que lo proporcionan.

\section{4. ¿Cuales son los costos asociados a la evolución de la conceptualización del servicio público?}

Para abordar esta cuestión, hemos adoptado un abordaje metodológico de cariz cuantitativo, realizado a partir de la aplicación del INSAT - INquérito SAúde e Trabalho (Encuesta Salud y Trabajo) (Barros-Duarte, Cunha \& Lacomblez, 2007) a una muestra de 161 conductores, de los cuales 158 eran hombres y 3 mujeres, integrados en cinco empresas, una del sector público (90 conductores de la muestra total) y las cuatro empresas restantes del sector privado (71 conductores de la muestra total), con el objetivo de analizar sus percepciones sobre las condiciones en las que ejercen su actividad de trabajo y sus efectos a nivel de la salud.

Los constreñimientos relativos a la extensión de la jornada de trabajo son identificados por casi todos los trabajadores participantes (82 conductores - $91.1 \%$ en la empresa pública, 69 conductores - el $97.2 \%$, en las empresas privadas). Pero si bien no existen diferencias significativas en este ítem relativas al tipo de empresa, los conductores de las empresas privadas declaran, casi cuatro veces más $(\mathrm{OR}=3.50)\left[{ }^{4}\right]$ exceder el horario normal de trabajo, en comparación con los conductores de la empresa pública. Esta es también una de las principales quejas de los trabajadores, expresando la tendencia de las empresas privadas de reducir el tiempo prescripto de realización de los recorridos, desfasándolo a menudo de las necesidades reales.

Sin embargo, estos resultados pueden parecer paradójicos con aquellos que señalan el hecho de que los conductores de las empresas privadas de la muestra revelan cerca de siete veces más probabilidades que los conductores de la empresa pública, que en la gestión de su actividad influyen sobre el ritmo o la velocidad de trabajo $(\mathrm{OR}=7.35)$, y 
que toman decisiones por sí mismos. Esto es visible, especialmente cuando aceleran o atrasan la circulación en función de la distancia a la que se encuentran los conductores de otras empresas que operan en la misma línea. En un contexto en el que el servicio público es atravesado por situaciones competitivas, preservar "la buena distancia" constituye la prioridad de los conductores, es decir, "no andar pegado" a los conductores de otras empresas que comparten el mismo recorrido, ya que esto equivale a un servicio "perdido" (posibilidad de no llevar pasajeros en este viaje).

Esto significa que la mayor libertad que aparentemente disponen los conductores de las empresas privadas para gestionar su actividad de servicio público, se les concede en favor de la consecución de los objetivos de rentabilidad. Y, debido a una deficiente regulación del servicio prestado, se transfiere a estos trabajadores la responsabilidad de tomar decisiones, que requieren un uso de sí (Schwartz, 2000b), en un intento de satisfacer el interés de la propia empresa, sin comprometer la calidad del servicio prestado al cliente (Orban, 2004).

Sin embargo, estos intentos no siempre son exitosos. Un ejemplo de esto es el hecho de que estos conductores se refieren a estar expuestos al riesgo de agresión verbal, con una probabilidad cerca de 15 veces mayor $(\mathrm{OR}=14.72)$ a los conductores de la empresa pública.

No se trata, obviamente, tal como se refiere Schwartz y Durrive (2003), de sostener aquí la eliminación de los valores de mercado, pero sí considerar los costos de los intentos de reequilibrar las habituales relaciones de subordinación entre diferentes valores en la actividad.

\section{Segundo estudio de caso : diseño de una red de transporte y actividad de los conductores en un contexto predominantemente rural}

Hablar de los costos inherentes a la evolución de la noción de servicio público, a partir de un análisis basado en la comparación entre lo "público" y lo "privado" correría el riesgo de llevarnos a una situación de impasse (Orban, 2004). Es imprescindible tener en cuenta también otras categorías de análisis, en la reflexión sobre los costos individuales y colectivos de la evolución de la noción de servicio público.

29 Ejemplo de esto es el concepto de territorio, a menudo subestimado en los discursos sobre la movilidad, a pesar de ser un elemento diferenciador de las formas de movilidad producidas.

Nuestro segundo estudio de caso se desarrolló en el ámbito del proyecto de rediseño de la red de transporte público de ómnibus de un área concedida de la región centro de Portugal, tipificada como "predominantemente rural" (INE, 2009). La solicitud de intervención en el rediseño de la red de transportes de ómnibus, fue protagonizada por la empresa privada que tiene el monopolio de las concesiones de los recorridos en el municipio, en colaboración con la Cámara Municipal (responsables por el pago de "indemnizaciones compensatorias" por la prestación del servicio de transporte público en el Municipio) y dirigido a un equipo de diseñadores de la movilidad (constituida en su mayoría por ingenieros, además de geógrafos y matemáticos). 
31 El territorio en cuestión comprende un área extensa, con un nivel de densidad poblacional considerado bajo (aproximadamente 40 habitantes por $\mathrm{km} 2$ ), lo que significa una limitación importante en una intervención dirigida a mejorar las condiciones de movilidad y asegurar la viabilidad económica de los servicios prestados. En este caso concreto se hace visible el dimensionamiento de valores del bien común traducidos en garantizar que el derecho a la movilidad ocurra de forma privilegiada en lugares donde la operacionalización de este derecho pueda conciliarse con la sostenibilidad económica de los servicios prestados, es decir, lugares donde existe una mayor demanda. E esto incluso cuando se define como obligatoria la prestación de servicios de transporte, como en el transporte escolar (Decreto-Ley n $\cong 299$ /84, de 5 de septiembre). De hecho, fue por esta razón que en el proyecto de rediseño de la red de movilidad se haya tenido una especial atención a las necesidades de los estudiantes, ya que son los principales usuarios del transporte público y los que mantienen, durante todo el año, un patrón de uso regular.

En términos metodológicos, se hicieron observaciones de la actividad de los diseñadores y los conductores en su contexto real y en cada una de las líneas que conforman la red, complementadas con entrevistas sobre las necesidades específicas que, desde su punto de vista, el diseño de la nueva red debería ser capaz de satisfacer.

33 Antes de la intervención, la prestación de servicios de transporte público se limitaba a sólo dos viajes (un viaje por la mañana y otro en la hora pico de la tarde) ; durante el período escolar ; y en los días hábiles.

Los resultados obtenidos visibilizan la ausencia de un espacio compartido entre los diseñadores y los conductores para la discusión sobre la actividad concreta de éstos, sus condiciones de trabajo, las dificultades y las estrategias que desarrollan para superarlas, y los compromisos que tienen que construir para garantizar el transporte a los que están más alejados de los recorridos de los ómnibus. Desde la perspectiva de los conductores no siempre serían respaldadas las decisiones de "corte" de algunos tramos de los recorridos teniendo como referencia "calcular" el número de pasajeros, teniendo en cuenta que estos fueron hechos en forma discontinua en el tiempo. Los diseñadores, por su parte, asumen las variaciones relativas a lo que habitualmente sucede, o lo que se prescribe, como " irregularidades", si bien que constituyen alternativas esenciales para satisfacer las necesidades de los usuarios y, en general, para preservar el derecho a la movilidad de una parte de la población. Tal es el caso, por ejemplo, de realizar más paradas de las previstas, o la introducción de desvíos en el recorrido, para llevar a los que están más lejos, con el consiguiente adelanto del horario de inicio del recorrido, para asegurar que a pesar de estos desvíos, los horarios de los ómnibus sean compatibles con el horario escolar.

35 Si los transportes públicos son garantizados en un espacio delimitado, basados en la intención de los que definen su trazado, al mismo tiempo, la actividad concreta y cotidiana en este espacio lo actualiza - respondiendo a necesidades específicas, como hacer una parada en lugares no previstos y otorgando de otros valores las relaciones en este espacio. Sin embargo, a pesar que la contribución de los conductores sea indispensable en la respuesta a las necesidades que el sistema de transporte no es capaz de satisfacer, ellos se presentan en este proyecto de rediseño más como "(...) objeto de estudio (...) [que] como protagonistas del trabajo de investigación" (Clot, 1981, traducción libre). 
36 No fueron, sin embargo, objeto de estudio sus condiciones de trabajo y empleo, entendidas como una cuestión extrínseca al proyecto, a pesar que la mejora en las condiciones de transporte derivaran de las posibilidades introducidas por la actividad de estos trabajadores. En un contexto poco densificado como este, la situación laboral de los conductores está marcada por la precariedad de los contratos de trabajo, a menudo a tiempo parcial, debido a que la operación de la red se limita a dos circulaciones por día, en cada línea, durante el periodo escolar. No dejan, por lo tanto de perpetuarse en silencio las desigualdades de sus condiciones de trabajo y empleo, en comparación con aquellos que ejercen la misma actividad en territorios considerados más atractivos...

\section{Conclusiones}

37 La tensión entre el "concepto" de servicio público y la "realidad" que se observa en los resultados presentados, vuelve visible cómo el debate sobre este concepto estructurante en la vida colectiva no podrá limitarse a los principios que conceptualmente le son intrínsecos, manteniendo en la penumbra la forma en como este es concretamente concebido y organizado para satisfacer las necesidades que están en su origen. Y si se tratara de una cuestión emergente a la que se necesita dar respuesta, también sabemos que va más allá de los límites de este estudio. Las condiciones de trabajo asociadas a la prestación de un servicio público y las opciones de organización del trabajo que lo configuran son, después de todo, un problema de la sociedad. La contribución de esta tesis se traduce en apoyar una mayor atención a los conflictos que se dejan entrever con la evolución de las normas que regulan su prestación, dando lugar en algunos casos a una usurpación de los valores que le son identitarios (Linhart, 2009). El hecho de no convocar el punto de vista del trabajo, anclado en sus especificidades territoriales, vuelve ilusoria la idea de que es posible definir "buenas prácticas" en materia de políticas de transporte, diseñadas en "desadherencia", y por eso tan universales y transferibles. ¿Cómo garantizar, entonces, el derecho a la movilidad y el derecho al territorio ?

38 Y si hablamos de derechos, sigue siendo paradójico que, en Portugal, los conductores en este caso del sector público hayan visto recientemente promulgada la pérdida del derecho al transporte gratuito (en el caso específico de los trabajadores jubilados, y recomendada su limitación para los trabajadores activos), cuando se les asignó esta compensación en lugar de los aumentos salariales. Es necesario, pues, que el espacio público en el que ejercen su actividad sea también el escenario de discusión del desmanteliamento de este derecho, con la expectativa de que la actual coyuntura no impida la movilización para el hacer... 


\section{BIBLIOGRAFÍA}

Ascher, F. (2010). Les nouveaux principes de l'urbanisme. La Tour d'Aigues, France : Éditions de l'Aube.

Barros-Duarte, C., Cunha, L., \& Lacomblez, M. (2007). INSAT : uma proposta metodológica para análise dos efeitos das condições de trabalho sobre a saúde, Laboreal, 3(2), 54-62.

Castells, M. (2000). A questão urbana (A. Caetano Trad.). São Paulo, Brasil : Edições Paz e Terra. Chevallier, J. (1987). Le service public. Paris, France : PUF.

Clot, Y. (1981). Présentation. In I. Oddone, A. Re \& G. Briante (Eds.), Redécouvrir l'expérience ouvrière. Vers une autre psychologie du travail ? (I. Barsotti \& M.-L. Barsotti Trad.) (pp. 7-13). Paris : Éd. Sociales.

Commission des Communautés Européennes (2000). Règlement du Parlement Européen et du Conseil relatif à l'action des États membres en matière d'exigence de service public et à l'attribution de contrats de service public dans le domaine des transports de voyageurs par chemin de fer, par route et par voie navigable, COM(2000) 7 final. Bruxelles, 26.7.2000.

Cunha, L., \& Lacomblez, M. (2006). Mudanças no sector dos transportes em Portugal : que caminhos para a actividade de serviço público e para a preservação do interesse geral ? Laboreal, 2, (2), 26-37. http://laboreal.up.pt/revista/artigo.php?id=37t45nSU54711227854127:811

Cunha, L., \& Lacomblez, M. (2007). Market and regulation of general interest in the passenger land transport sector: a debate renewed by drivers' activity. @ctivités, 4 (1), pp. 141-148, http:// www.activites.org/v4n1/v4n1.pdf

Cunha, L., \& Lacomblez, M. (2010). La production d'un service public : ce que révèle l'activité de conducteurs de bus au Portugal. In H. Maksim, S. Vincent, C. Gallez \& V. Kaufmann (Dir.). L'action publique face à la mobilité (pp. 51-75). Paris : Éditions L'Harmattan.

Derrienic, F., Saurel-Cubizolles, M.-J., \& Monfort, C. (2003). Santé, conditions de travail et cessation d'activité des salariés âgés. Travail et Emploi, 96, 37-53.

Dreyfus, J-D. (1999). Service public et secteur public : association ou dissociation ? In H. Groud (Ed.), Mutations du service public et territoires, (pp. 11-18). Paris, France : Éditions L'Hartmattan.

Fernandes, J. (1996). Dicionário jurídico da Administração Pública. Lisboa, Portugal : Almedina.

Guèrin, F., Laville, A., Daniellou, F., Duraffourg, J., \& Kerguelen, A. (1991). Comprendre le travail pour le transformer. Paris, France : Éditions ANACT.

Instituto Nacional de Estatística (INE) (2009). Tipologia de áreas urbanas. Retirado de www.ine.pt Lannoy, P., \& Ramadier, T. (Eds.) (2007). La mobilité généralisée. Formes et valeurs de la mobilité quotidienne. Louvain-la-Neuve, Belgique : Academia Bruylant.

Le Van-Lemesle, L. (2005). Cauwès et Colson, le juriste et l'ingénieur : une ou deux conceptions du service public ? Revue d'histoire moderne et contemporaine, 3 (52-53), 75-87.

Linhart, D. (2009). Comment l'entreprise usurpe les valeurs du service public. Le Monde diplomatique, 666, 20.

Mincke, C., \& Montulet, B. (2010). L'idéologie mobilitaire, Politique, 64. Retirado de http:// politique.eu.org 
Mom, G. (2009). Inventer et établir l'Histoire de la mobilité : aux origines d'un changement de paradigme. In M. Flonneau \& V. Guigueno (Eds.), De l'histoire des transports à l'histoire de la mobilité? (pp. 25-39). Rennes, France : Presses Universitaires de Rennes.

Némery, J.-C., \& Routier, J.-C. (1999). Avant-propos. In H. Groud (Ed.), Mutations du service public et territoires, (pp. 7-10). Paris, France : L'Hartmattan.

Orban, E. (2004). Service public! Individu, marché et intérêt public. Paris, France : Éditions Syllepse.

Scheller, L., Cunha, L., Nogueira, S., \& Lacomblez, M. (2013). Le temps des conductrices de bus en France et au Portugal. Travail, genre et sociétés, n.ำ 29, 69-86.

Schwartz, Y. (2000a). Le paradigme ergologique ou un métier de philosophe. Toulouse, France : Octarès Éditions.

Schwartz, Y. (2000b). Trabalho e uso de si. Pró-posições, 1, 5 (32), 34-50.

Schwartz, Y., \& Durrive, L. (Eds.) (2003). Travail \& Ergologie. Entretiens sur l'activité humaine. Toulouse, France : Octarès Éditions.

Steiner, P. (2007). La sociologie économique. Paris, France : Éditions La Découverte.

Urry, J. (2004). The ‘System' of Automobility. Theory, Culture \& Society, 21, 25-39.

\section{NOTAS}

1. El "encastramiento" es un concepto desarrollado por Karl Polanyi y retomado por la "nueva sociología económica" (Steiner, 2007), que sustenta la comprensión de los factores sociales que sostienen el funcionamiento de las economías de mercado, asumiendo que lo económico esta "encastrado" en lo social.

2. Aunque en la tradición de la ergonomía y la psicología del trabajo de la actividad es común el uso de este término en el sentido de "cualquier persona que realice una actividad profesional, sean cuales fueren sus características" (Guèrin, Laville, Daniellou, Duraffourg \& Kerguelén, 1991, p.15, traducción libre), mantenemos aquí el uso de este concepto, ya que es parte del "lenguaje operativo" del sector de actividad en análisis. La definición que figura en este contexto particular, y que aquí adoptamos corresponde a una empresa de derecho público o privado a la que se le asigna la responsabilidad de la prestación de servicios de transporte público de pasajeros en áreas concesionadas de explotación de líneas por el gobierno central o local (Commission des Communautés Européennes, 2000).

3. La venta de boletos y las tarifas de las tres empresas también sufrieron cambios, siendo igual para los tres operadores, que pasaron a compartir el sistema "Andante". En este sentido, el cliente siempre puede tomar el primer ómnibus, independientemente de la empresa de transportes que se trate.

4. El odds ratio (OR) es una medida del riesgo relativo de estar expuesto a una situación de trabajo en particular, para un determinado factor o grupo (por ejemplo, ser conductor en una empresa privada), en comparación con la clase de referencia definida (ser conductor de una empresa pública). $\mathrm{Si} O \mathrm{R}>1$, se dice que el factor asociado constituye un posible riesgo de exposición, mientras que en el caso contrario, $\mathrm{OR}<1$, el factor puede tener un efecto protector contra la exposición (Derrienic, Saurel-Cubizolles \& Monfort, 2003). 


\section{AUTORES}

\section{LILIANA CUNHA}

Centro de Psicologia da Universidade do Porto, Centro de Estudos em Desenvolvimento Humano, Faculdade de Educação e Psicologia, Centro Regional do Porto da Universidade Católica

Portuguesa, Rua Diogo Botelho, 1327, 4169-004 Porto

lcunha@porto.ucp.pt 